\title{
Excited-state pair-density-functional theory
}

\author{
Á. Nagy \\ Department of Theoretical Physics, University of Debrecen, H-4010 Debrecen, Hungary
}

(Received 12 June 2014; published 11 August 2014)

\begin{abstract}
The theory of pair density is extended to excited states. The theory of a single excited state is generalized for the pair density. A two-particle equation is derived for the square root of the pair density of the given excited state. An expression for the effective potential of this equation is presented.
\end{abstract}

DOI: 10.1103/PhysRevA.90.022505

PACS number(s): $31.15 . \mathrm{E}-$

\section{INTRODUCTION}

Recently there has been a growing interest in the theory of pair density, where the fundamental variable is not the electron density, but the pair density. Ziesche [1] extended the Hohenberg-Kohn theorems [2] for the pair density. Another approach was presented by Gonis et al. [3]. It was shown by the present author $[4,5]$ that in the ground state the pair density can be determined by solving a single auxiliary equation of a two-particle problem. Thus, the problem of an arbitrary system can be reduced to a two-particle problem. A similar result was later presented by Furche [6].

In this paper the theory of pair density is extended to excited states. In density-functional theory there are several timeindependent approaches to treat excited states: the ensemble theories [7-9] and the theories for a single excited state [10-15]. Here the approach of $[12,13]$ of a single excited state is generalized for the pair density. The paper is organized as follows. In Sec. II the previous theory is summarized. Section III presents the generalization of the theory for the pair density. In Sec. IV a two-particle equation is derived for the square root of the pair density of the given excited state. The last section is devoted to discussion.

\section{THEORY FOR A SINGLE EXCITED STATE}

First the theory is summarized in order that the extension of the theory of pair density to excited states can be followed more easily. Consider the Hamiltonian of interest,

$$
\hat{H}=\hat{T}+\hat{V}_{e e}+\sum_{i=1}^{N} v\left(\mathbf{r}_{i}\right) .
$$

The kinetic energy and the electron-electron energy operators have the form

$$
\hat{T}=\sum_{i=1}^{N}\left(-\frac{1}{2} \nabla_{i}^{2}\right)
$$

and

$$
\hat{V}_{e e}=\sum_{i<j}^{N} \frac{1}{\left|\mathbf{r}_{i}-\mathbf{r}_{j}\right|},
$$

where $v(\mathbf{r})$ is a local external potential.

The ground-state energy is given by the variational principle,

$$
E_{0}=\min _{\Psi}\langle\Psi|\hat{H}| \Psi\rangle
$$

In the constraint search approach $[16,17]$ the minimization can be done in two steps,

$$
E_{0}=\min _{\varrho} \min _{\Psi \rightarrow \varrho}\langle\Psi|\hat{H}| \Psi\rangle .
$$

The inner step minimization for all the antisymmetric wave functions providing the density $\varrho$ leads to the energy functional $E$,

$$
E[\varrho]=\min _{\Psi \rightarrow \varrho}\langle\Psi|\hat{H}| \Psi\rangle .
$$

The ground-state energy can be written as

$$
E_{0}=\min _{\varrho}\left\{\int v(\mathbf{r}) \varrho(\mathbf{r}) d \mathbf{r}+F[\varrho]\right\},
$$

where the universal functional $F$ is defined as

$$
F[\varrho]=\min _{\Psi \rightarrow \varrho}\left\langle\Psi\left|\hat{T}+\hat{V}_{e e}\right| \Psi\right\rangle .
$$

Then we apply the variational principle for the excited state $i$,

$$
E_{i}=\min _{\varrho} \min _{\substack{\Psi \rightarrow \varrho \\ \Psi \perp \Psi_{0}, \ldots, \Psi_{i-1}}}\langle\Psi|\hat{H}| \Psi\rangle .
$$

The inner minimization defines the energy density functional for the $i$ th excited state:

$$
E_{i}[\varrho, v]=\min _{\substack{\Psi \rightarrow \varrho \\ \Psi \perp \Psi_{0}, \ldots, \Psi_{i-1}}}\langle\Psi|\hat{H}| \Psi\rangle .
$$

The minimization is over all wave functions that are orthogonal to the first $i-1$ states of $\hat{H}$ and simultaneously gives the density $\varrho$.

Note that for $i=0$ the energy density functional is the ground-state energy functional $E[\varrho]$ [Eq. (6)]. Here a nondegenerate case is treated. (The extension to degenerate states can be done utilizing the subspace densities [13].)

We can rewrite this procedure as

$$
\begin{aligned}
E_{i} & =\min _{\varrho}\left\{\int v(\mathbf{r}) \varrho(\mathbf{r}) d \mathbf{r}+F_{i}\left[\varrho, \varrho_{0}\right]\right\} \\
& =\int v(\mathbf{r}) \varrho_{i}(\mathbf{r}) d \mathbf{r}+F_{i}\left[\varrho_{i}, \varrho_{0}\right],
\end{aligned}
$$

where the functional $F_{i}\left[\varrho, \varrho_{0}\right]$ is defined as

$$
\begin{aligned}
F_{i}\left[\varrho, \varrho_{0}\right]= & \min _{\substack{\Psi \rightarrow n \\
\Psi \perp \Psi_{0}, \ldots, \Psi_{i-1}}}\left\langle\Psi\left|\hat{T}+\hat{V}_{e e}\right| \Psi\right\rangle \\
= & \left\langle\Psi\left[\varrho, \varrho_{0}\right]\left|\hat{T}+\hat{V}_{e e}\right| \Psi\left[\varrho, \varrho_{0}\right]\right\rangle
\end{aligned}
$$


In Eq. (12) $\Psi$ yields $\varrho$ and is orthogonal to the first $i-1$ state of the Hamiltonian for which $\varrho_{0}$ is the ground-state density. Here this Hamiltonian is the $\hat{H}$ in Eq. (4). We mention in passing that the Kohn-Sham theory was also extended to excited states $[12,13]$. Note that instead of the ground-state electron density $\varrho_{0}$, we could use the external potential $v$ or any ground-state Kohn-Sham orbital, etc. Thus, we could use $F_{i}[\varrho, v]$.

Görling also formalized an excited-state density-functional theory using the stationary principle [18]. In a recent paper Ayers and Levy [19] gave Görling's excited-state functional a firm theoretical foundation. They also proved that Görling's functional is a restriction of the functional (12) to those external potentials for which it is stationary.

One needs approximate exchange-correlation potentials to perform excited-state calculations. Local self-interactionfree approximate exchange-correlation potentials have been proposed for this purpose [20]. Orbital-dependent functionals (optimized potential method (OPM) [21] and the KriegerLi-Iafrate (KLI) method [22]) were also generalized and tested [10,12,13,23,24]. Glushkov and Levy [25] presented an OPM algorithm that takes the necessary orthogonality constraints to lower states into account.

The standard Hohenberg-Kohn theorems for a single excited-state density do not exist [26-28]. Gaudoin and Burke [27] and Sahni, Slamet, and Pan [28] performed numerical calculations and presented cases where a given excitedstate density corresponds to several different "Kohn-Sham" potentials. Samal, Harbola, and Holas [29] argued that the density-to-potential map can be fixed uniquely by the criterion given in [12]. In another paper, Samal and Harbola [30] proposed a different criterion. Harbola and co-workers also developed a local spin density functional approximation by splitting $k$ space and obtained accurate excitation energies [31-37].

\section{GENERALIZATION OF THE THEORY FOR THE PAIR DENSITY}

The second-order reduced density matrix is defined as

$$
\begin{aligned}
n_{2}\left(\mathbf{x}_{1}, \mathbf{x}_{2} ; \mathbf{x}_{1}^{\prime}, \mathbf{x}_{2}^{\prime}\right)= & \frac{N(N-1)}{2} \int \Psi\left(\mathbf{x}_{1}, \mathbf{x}_{2}, \mathbf{x}_{3}, \ldots, \mathbf{x}_{N}\right) \Psi^{*} \\
& \times\left(\mathbf{x}_{1}^{\prime}, \mathbf{x}_{2}^{\prime}, \mathbf{x}_{3}, \ldots, \mathbf{x}_{N}\right) d \mathbf{x}_{3}, \ldots, d \mathbf{x}_{N},
\end{aligned}
$$

where $\mathbf{x}_{i}$ stands for the spatial and the spin coordinates: $\mathbf{r}_{i}, \sigma_{i}$. The diagonal of the spin-independent second-order density matrix is the pair density:

$$
n\left(\mathbf{r}_{1}, \mathbf{r}_{2}\right)=\int n_{2}\left(\mathbf{x}_{1}, \mathbf{x}_{2} ; \mathbf{x}_{1}, \mathbf{x}_{2}\right) d \sigma_{1} d \sigma_{2} .
$$

Consider first the ground state. The constrained search method can be applied for the pair density $n$,

$$
E=\min _{n} \min _{\Psi \rightarrow n}\langle\Psi|\hat{H}| \Psi\rangle,
$$

where the search goes for all antisymmetric wave functions $\Psi$, which yield the given $n$. We can define the universal functional $T$ as

$$
T[n]=\min _{\Psi \rightarrow n}\langle\Psi|\hat{T}| \Psi\rangle .
$$

Consequently, the ground-state energy can also be written as

$$
\begin{aligned}
E= & \min _{n}\left\{\int \frac{n\left(\mathbf{r}_{1}, \mathbf{r}_{2}\right)}{\left|\mathbf{r}_{1}-\mathbf{r}_{2}\right|} d \mathbf{r}_{1} d \mathbf{r}_{2}\right. \\
& \left.+\frac{1}{N-1} \int u\left(\mathbf{r}_{1}, \mathbf{r}_{2}\right) n\left(\mathbf{r}_{1}, \mathbf{r}_{2}\right) d \mathbf{r}_{1} d \mathbf{r}_{2}+T[n]\right\} .
\end{aligned}
$$

The factor $1 /(N-1)$ comes from the normalization of $n$. The external potential is conveniently written for the pair of particles as

$$
u\left(\mathbf{r}_{i}, \mathbf{r}_{j}\right)=v\left(\mathbf{r}_{i}\right)+v\left(\mathbf{r}_{j}\right) .
$$

Consider now the excited state $i$. The constrained search method is applied again, now for the pair density $n$ :

$$
E_{i}=\min _{n} \min _{\substack{\Psi \rightarrow n \\ \Psi \perp \Psi_{0}, \ldots, \Psi_{i-1}}}\langle\Psi|\hat{H}| \Psi\rangle .
$$

The search is for all antisymmetric wave functions $\Psi$ that are orthogonal to the first $i-1$ states of $\hat{H}$ and simultaneously gives the trial pair density, $n$. We can define the functional $T_{i}[n, u]$ as

$$
T_{i}[n, u]=\min _{\substack{\Psi \rightarrow n \\ \Psi \perp \Psi_{0}, \ldots, \Psi_{i-1}}}\langle\Psi|\hat{T}| \Psi\rangle .
$$

The energy functional then takes the form

$$
\begin{aligned}
E_{i}[n, u]= & \int \frac{n\left(\mathbf{r}_{1}, \mathbf{r}_{2}\right)}{\left|\mathbf{r}_{1}-\mathbf{r}_{2}\right|} d \mathbf{r}_{1} d \mathbf{r}_{2} \\
& +\frac{1}{N-1} \int u\left(\mathbf{r}_{1}, \mathbf{r}_{2}\right) n\left(\mathbf{r}_{1}, \mathbf{r}_{2}\right) d \mathbf{r}_{1} d \mathbf{r}_{2}+T_{i}[n, u] .
\end{aligned}
$$

The energy functional $E_{i}[n, u]$ has important properties.

Theorem 1. The exact energy of the $i$ th excited state is a lower bound to $E_{i}[n, u]$,

$$
E_{i} \leqslant E_{i}[n, u]
$$

with equality holding only when $n=n_{i}$ is the exact density of the $i$ th excited state.

Proof. Let $\Psi_{\min }^{n}$ denote that antisymmetric wave function that satisfies [Eq. (20)]

$$
T_{i}[n, u]=\left\langle\Psi_{\min }^{n}|\hat{T}| \Psi_{\min }^{n}\right\rangle .
$$

Adding terms corresponding to the electron-electron and the external term we obtain

$$
\begin{aligned}
\int \frac{n\left(\mathbf{r}_{1}, \mathbf{r}_{2}\right)}{\left|\mathbf{r}_{1}-\mathbf{r}_{2}\right|} d \mathbf{r}_{1} d \mathbf{r}_{2} \\
\quad+\frac{1}{N-1} \int u\left(\mathbf{r}_{1}, \mathbf{r}_{2}\right) n\left(\mathbf{r}_{1}, \mathbf{r}_{2}\right) d \mathbf{r}_{1} d \mathbf{r}_{2}+T_{i}[n, u] \\
=\int \frac{n\left(\mathbf{r}_{1}, \mathbf{r}_{2}\right)}{\left|\mathbf{r}_{1}-\mathbf{r}_{2}\right|} d \mathbf{r}_{1} d \mathbf{r}_{2} \\
\quad+\frac{1}{N-1} \int u\left(\mathbf{r}_{1}, \mathbf{r}_{2}\right) n\left(\mathbf{r}_{1}, \mathbf{r}_{2}\right) d \mathbf{r}_{1} d \mathbf{r}_{2}+\left\langle\Psi_{\min }^{n}|\hat{T}| \Psi_{\min }^{n}\right\rangle \\
=\left\langle\Psi_{\min }^{n}\left|\hat{V}_{e e}+\hat{V}+\hat{T}\right| \Psi_{\min }^{n}\right\rangle .
\end{aligned}
$$

The variational principle leads to the first part of the theorem:

$$
\left\langle\Psi_{\min }^{n}\left|\hat{V}_{e e}+\hat{V}+\hat{T}\right| \Psi_{\min }^{n}\right\rangle=E_{i}[n, u] \geqslant E_{i} .
$$


Denote $\Psi_{i}^{n_{i}}$ the exact wave function corresponding to the pair density $n_{i}$. Then

$$
T_{i}\left[n_{i}, u\right]=\left\langle\Psi_{i}^{n_{i}}|\hat{T}| \Psi_{i}^{n_{i}}\right\rangle .
$$

By the variational principle,

$$
E_{i} \leqslant\left\langle\Psi_{\min }^{n}\left|\hat{V}_{e e}+\hat{V}+\hat{T}\right| \Psi_{\min }^{n}\right\rangle .
$$

Therefore,

$$
\left\langle\Psi_{i}^{n_{i}}\left|\hat{V}_{e e}+\hat{V}+\hat{T}\right| \Psi_{i}^{n_{i}}\right\rangle \leqslant\left\langle\Psi_{\min }^{n}\left|\hat{V}_{e e}+\hat{V}+\hat{T}\right| \Psi_{\min }^{n}\right\rangle .
$$

It can also be written as

$$
\begin{aligned}
& \int \frac{n_{i}\left(\mathbf{r}_{1}, \mathbf{r}_{2}\right)}{\left|\mathbf{r}_{1}-\mathbf{r}_{2}\right|} d \mathbf{r}_{1} d \mathbf{r}_{2} \\
& \quad+\frac{1}{N-1} \int u\left(\mathbf{r}_{1}, \mathbf{r}_{2}\right) n_{i}\left(\mathbf{r}_{1}, \mathbf{r}_{2}\right) d \mathbf{r}_{1} d \mathbf{r}_{2}+\left\langle\Psi_{i}^{n_{i}}|\hat{T}| \Psi_{i}^{n_{i}}\right\rangle \\
& \leqslant \int \frac{n_{i}\left(\mathbf{r}_{1}, \mathbf{r}_{2}\right)}{\left|\mathbf{r}_{1}-\mathbf{r}_{2}\right|} d \mathbf{r}_{1} d \mathbf{r}_{2} \\
& \quad+\frac{1}{N-1} \int u\left(\mathbf{r}_{1}, \mathbf{r}_{2}\right) n_{i}\left(\mathbf{r}_{1}, \mathbf{r}_{2}\right) d \mathbf{r}_{1} d \mathbf{r}_{2}+\left\langle\Psi_{\min }^{n}|\hat{T}| \Psi_{\min }^{n}\right\rangle
\end{aligned}
$$

or

$$
\left\langle\Psi_{i}^{n_{i}}|\hat{T}| \Psi_{i}^{n_{i}}\right\rangle \leqslant\left\langle\Psi_{\min }^{n}|\hat{T}| \Psi_{\min }^{n}\right\rangle .
$$

On the other hand, the consequence of the definition of $\Psi_{\min }^{n}$ is

$$
\left\langle\Psi_{i}^{n_{i}}|\hat{T}| \Psi_{i}^{n_{i}}\right\rangle \geqslant\left\langle\Psi_{\min }^{n}|\hat{T}| \Psi_{\min }^{n}\right\rangle .
$$

Equations (30) and (31) can be true if and only if the equality holds that immediately leads to the validity of the second part of the theorem.

Denote the wave function that minimizes the energy functional $E_{i}\left[n, n_{0}\right]$ by $\Psi_{i}\left[n, n_{0}\right]$ :

$$
\Psi_{i}[n, u] \text { minimizes } E_{i}[n, u],
$$

Theorem 2. The pair density, the external potential, and the degree of excitation $i$ determines the wave function.

Proof. The constrained search described above gives to the wave function.

Theorems 1 and 2 can be considered a generalization of the Hohenberg-Kohn theorems for excited-state pair densities.

Note that the excited-state wave function $\Psi_{i}[n, u]$ is not just a functional of the excited-state pair density and $i$ but also a functional of the external potential $u$. It can be immediatelly seen from Eq. (21) as the functional $T_{i}[n, u]$ can be written as

$$
\begin{aligned}
T_{i}[n, u]= & E_{i}-\int \frac{n\left(\mathbf{r}_{1}, \mathbf{r}_{2}\right)}{\left|\mathbf{r}_{1}-\mathbf{r}_{2}\right|} d \mathbf{r}_{1} d \mathbf{r}_{2} \\
& -\frac{1}{N-1} \int u\left(\mathbf{r}_{1}, \mathbf{r}_{2}\right) n\left(\mathbf{r}_{1}, \mathbf{r}_{2}\right) d \mathbf{r}_{1} d \mathbf{r}_{2} .
\end{aligned}
$$

$T_{i}$ depends parametrically on the external potential through the constraint that the wave function should be orthogonal to the lower-lying eigenfunctions of the Hamiltonian. Therefore, unlike the ground-state Hohenberg-Kohn functional, $T_{i}$ is not a universal functional of the pair density.

Instead of the external potential, the ground-state pair density $n_{0}$ can also be selected. Applying $T_{i}[n, u]$ instead of
$T_{i}\left[n, n_{0}\right]$ has the advantage that the direct appearance of the external potential avoids the $v$-representability problem. A pair density is $v$-representable if it is the pair density associated with the $i$ th eigenfunction of a Hamiltonian of the form (1) with some external potential $v$. [Instead of $v$ it is convenient to use $u$ here according to Eq. (18).] If we have the functional $T_{i}[n, u]$ the $v$ representability is automatically fulfilled as $n$ is the pair density corresponding to the $i$ th eigenfunction of the Hamiltonian with external potential $u$.

Also, it is more natural to use the external potential as a variable than the ground-state density, as in calculations the external potential is the usual input. Supposing that the functional derivative of $T_{i}$ exists, Eq. (33) leads to the Euler equation,

$$
u\left(\mathbf{r}_{1}, \mathbf{r}_{2}\right)+\frac{1}{\left|\mathbf{r}_{1}-\mathbf{r}_{2}\right|}=-\frac{\delta T_{i}[n, u]}{\delta n},
$$

up to a constant. In the following section, the Euler equation is reformulated as a two-particle equation for the square root of the pair density.

\section{TWO-PARTICLE EQUATION FOR THE SQUARE ROOT OF THE PAIR DENSITY OF THE GIVEN EXCITED STATE}

It was shown $[4,5,38,39]$ that the ground-state problem of an arbitrary system can be reduced to a two-particle problem, that is, only a single auxiliary equation of a two-particle system should be solved. Earlier, the method of Levy, Perdew, and Sahni [40] was generalized to derive this two-particle equation and an expression for the effective potential was obtained $[6,41]$. The same method can be applied for excited states.

The Schrödinger equation has the form

$$
\hat{H}(\bar{N}) \Psi_{i}(\bar{N})=E_{i}^{N} \Psi_{i}(\bar{N}),
$$

where $\bar{M}$ signifies the coordinates of electrons $1, \ldots, M$. Take the following partition of the Hamiltonian of the $N$-electron system,

$\hat{H}(\bar{N})=\hat{H}(N, N-1)+\hat{H}(\overline{N-2})+\sum_{j=1}^{N-2}\left(\frac{1}{r_{j N}}+\frac{1}{r_{j N-1}}\right)$,

where

$$
\begin{aligned}
\hat{H}(N, N-1)= & -\frac{1}{2} \nabla_{N}^{2}-\frac{1}{2} \nabla_{N-1}^{2}+v\left(\mathbf{r}_{N}\right)+v\left(\mathbf{r}_{N-1}\right) \\
& +\frac{1}{r_{N N-1}}
\end{aligned}
$$

and

$$
\hat{H}(\overline{N-2})=\sum_{j=1}^{N-2}\left[-\frac{1}{2} \nabla_{j}^{2}+v\left(\mathbf{r}_{j}\right)\right]+\sum_{j<k}^{N-2} \frac{1}{r_{j k}} .
$$

$v(\mathbf{r})$ is the external potential and $r_{j k}=\left|\mathbf{r}_{j}-\mathbf{r}_{k}\right|$. Introduce a function $\Phi_{i}(\bar{N})$ as

$$
\Phi_{i}(\overline{N-2} ; N-1, N)=\left[\frac{N(N-1)}{2}\right]^{1 / 2} \frac{\Psi_{i}(\bar{N})}{n_{i}^{1 / 2}\left(\mathbf{r}_{N-1}, \mathbf{r}_{N}\right)}
$$


$\Phi_{i}(\overline{N-2} ; N-1, N)$ is antisymmetric in electrons $1, \ldots, N-$ 2 and it depends parametrically on the variables of electrons $N-1$ and $N$. It has the property that

$$
\int\left|\Phi_{i}(\overline{N-2} ; N-1, N)\right|^{2} d(\overline{N-2})=1
$$

for any $\mathbf{r}_{N-1}$ and $\mathbf{r}_{N}$. It can be proved utilizing Eqs. (13), (14), and (39). Subtracting $E_{0}^{N-2} \Psi_{i}(\bar{N})$ from both sides of Eq. (35), multiplying by $\Phi_{i}(\overline{N-2} ; N-1, N)^{*}$, and integrating over all the coordinates of electrons $1, \ldots, N-2$ and spin coordinates of electrons $N-1, N$, the effective two-particle equation

$$
\left[-\frac{1}{2} \nabla_{N}^{2}-\frac{1}{2} \nabla_{N-1}^{2}+v\left(\mathbf{r}_{N}\right)+v\left(\mathbf{r}_{N-1}\right)+\frac{1}{r_{N N-1}}+\tilde{v}_{i}^{\mathrm{eff}}\left(\mathbf{r}_{N-1}, \mathbf{r}_{N}\right)\right] n_{i}^{1 / 2}\left(\mathbf{r}_{N-1}, \mathbf{r}_{N}\right)=\mu_{i} n_{i}^{1 / 2}\left(\mathbf{r}_{N-1}, \mathbf{r}_{N}\right)
$$

is obtained, where $E_{0}^{N-2}$ is the total ground-state energy of the $N$-2-electron system (after removing two electrons from the $N$-electron system) and

$$
\mu_{i}=E_{i}^{N}-E_{0}^{N-2}
$$

The effective potential takes the form

$$
\begin{aligned}
\tilde{v}_{i}^{\text {eff }}\left(\mathbf{r}_{N-1}, \mathbf{r}_{N}\right)= & \left\langle\Phi_{i}(\overline{N-2} ; N-1, N)\left|\hat{H}(\overline{N-2})-E_{0}^{N-2}\right| \Phi_{i}(\overline{N-2} ; N-1, N)\right\rangle \\
& +\frac{1}{2}\left[\left\langle\left|\nabla_{N} \Phi_{i}(\overline{N-2} ; N-1, N)\right|^{2}\right\rangle+\left\langle\left|\nabla_{N-1} \Phi_{i}(\overline{N-2} ; N-1, N)\right|^{2}\right\rangle\right] \\
& +\frac{6}{N(N-1)}\left[\int \frac{\tilde{n}_{i}\left(\mathbf{r}_{N-2}, \mathbf{r}_{N-1}, \mathbf{r}_{N}\right)}{r_{N-2, N-1}} d \mathbf{r}_{N-2}+\int \frac{\tilde{n}_{i}\left(\mathbf{r}_{N-2}, \mathbf{r}_{N-1}, \mathbf{r}_{N}\right)}{r_{N-2, N}} d \mathbf{r}_{N-2}\right],
\end{aligned}
$$

where $\tilde{n}_{i}\left(\mathbf{r}_{N-2}, \mathbf{r}_{N-1}, \mathbf{r}_{N}\right)$ is the density of that $\Phi_{i}(\overline{N-2} ; N-1, N)$ associated with electron $N-1$ at point $\mathbf{r}_{N-1}$ and electron $N$ at point $\mathbf{r}_{N}$. As no term on the right-hand side of Eq. (43) can ever be negative,

$$
\tilde{v}_{i}^{\text {eff }} \geqslant 0 \text {. }
$$

The asymptotic behavior of the effective potential $\tilde{v}_{i}^{\text {eff }}$ can be seen from the long-range form of the pair density [42]:

$$
n_{i}\left(\mathbf{r}_{N-1}, \mathbf{r}_{N}\right) \rightarrow e^{-2 \alpha_{i} r_{N-1}} e^{-2 \alpha_{i} r_{N}},
$$

where

$$
\alpha_{i}=\left[-\mu_{i}+\tilde{v}_{i}^{\text {eff }}(\infty, \infty)\right]^{1 / 2} .
$$

The inequality (44) leads to

$$
\alpha_{i} \geqslant \sqrt{-\mu_{i}} .
$$

Consider the system obtained after removing two electrons. The eigenstates of the Hamiltonian $\hat{H}(\overline{N-2})$ [Eq. (38)] are denoted by $\Psi_{l}(\overline{N-2})$. After multiplying Eq. (35) by $\Psi_{l}^{*}(\overline{N-2})$, integrating over the coordinates of $N-2$ electrons and using Eqs. (36)-(39) we arrive at

$$
\begin{aligned}
& {\left[-\frac{1}{2} \nabla_{N-1}^{2}-\frac{1}{2} \nabla_{N}^{2}+v\left(\mathbf{r}_{N-1}\right)+v\left(\mathbf{r}_{N}\right)+\frac{1}{r_{N-1, N}}-\varepsilon_{i}^{l}\right] g_{i}^{l}\left(\mathbf{r}_{N-1}, \mathbf{r}_{N}\right)} \\
& \quad=-(N-2) n^{1 / 2}\left(\mathbf{r}_{N-1}, \mathbf{r}_{N}\right)\left\langle\Psi_{l}(\overline{N-2})\left(\frac{1}{r_{1, N-1}}+\frac{1}{r_{1, N}}\right) \Phi_{i}(\overline{N-2} ; N-1, N)\right\rangle,
\end{aligned}
$$

where

$$
\varepsilon_{i}^{l}=E_{i}^{N}-E_{l}^{N-2},
$$

$E_{l}^{N-2}$ is the eigenvalue corresponding to the eigenfunction $\Psi_{l}(\overline{N-2})$, and

$$
\begin{aligned}
g_{i}^{l}\left(\mathbf{r}_{N-1}, \mathbf{r}_{N}\right)= & n_{i}^{1 / 2}\left(\mathbf{r}_{N-1}, \mathbf{r}_{N}\right) \\
& \times\left\langle\Psi_{l}^{*}(\overline{N-2}) \mid \Phi_{i}(\overline{N-2} ; N-1, N)\right\rangle .
\end{aligned}
$$

If $l=0$,

$$
\begin{aligned}
g_{i}^{0}\left(\mathbf{r}_{N-1}, \mathbf{r}_{N}\right)= & n_{i}^{1 / 2}\left(\mathbf{r}_{N-1}, \mathbf{r}_{N}\right)\left\langle\Psi_{0}^{*}(\overline{N-2})\right| \\
& \left.\times \Phi_{i}(\overline{N-2} ; N-1, N)\right\rangle .
\end{aligned}
$$

If $\quad r_{N-1} \rightarrow \infty \quad$ and $\quad r_{N} \rightarrow \infty, \quad g_{i}^{0}\left(\mathbf{r}_{N-1}, \mathbf{r}_{N}\right) \rightarrow$ $e^{-\sqrt{-\mu_{i}} r_{N-1}} e^{-\sqrt{-\mu_{i}} r_{N}}$ as $\varepsilon_{i}^{0}=\mu_{i}$. Taking into account the asymptotic form (45) of the pair density and the Schwartz inequality,

$$
\left|\left\langle\Psi_{0}^{*}(\overline{N-2}) \mid \Phi_{i}(\overline{N-2} ; N-1, N)\right\rangle\right| \leqslant 1,
$$

Eq. (51) provides that

$$
\alpha_{i} \leqslant \sqrt{-\mu_{i}}
$$

[There might be an exception: the case when symmetry induces that $\left\langle\Psi_{0}^{*}(\overline{N-2}) \Phi_{i}(\overline{N-2} ; N-1, N)\right\rangle=0$. If this is true, the state $\Psi_{0}^{*}(\overline{N-2})$ is called "inaccessible".] 
As both the inequalities (47) and (53) should hold simultaneously, we arrive at the equality

$$
\alpha_{i}=\sqrt{-\mu_{i}}
$$

Then the asymptotic form

$$
\tilde{v}_{i}^{\text {eff }}(\infty, \infty)=0
$$

arises from Eq. (46). Another consequence is that $\Phi_{i}(\overline{N-2} ; N-1, N)$ asymptotically collapses to the eigenfunction $\Psi_{0}(\overline{N-2})$. Equations (39) and (43) show that $\Phi_{i}(\overline{N-2})$ asymptotically cannot depend on the coordinates $\mathbf{r}_{N-1}$ and $\mathbf{r}_{N}$. Equation (43) leads to the asymptotic form of the effective potential:

$$
\tilde{v}_{i}^{\text {eff }} \rightarrow \frac{N-2}{r_{N-1}}+\frac{N-2}{r_{N}} .
$$

\section{DISCUSSION}

In the ground-state two-particle equation the Pauli potential was introduced. Now, Eq. (41) is written in a similar form,

$$
\begin{aligned}
& {\left[-\frac{1}{2} \nabla_{N-1}^{2}-\frac{1}{2} \nabla_{N}^{2}+v\left(\mathbf{r}_{N-1}\right)+v\left(\mathbf{r}_{N}\right)\right.} \\
& \left.\quad+\frac{N-1}{r_{N-1, N}}+v_{i}^{P}\left(\mathbf{r}_{N-1}, \mathbf{r}_{N}\right)\right] n_{i}^{1 / 2}\left(\mathbf{r}_{N-1}, \mathbf{r}_{N}\right) \\
& =\mu_{i} n_{i}^{1 / 2}\left(\mathbf{r}_{N-1}, \mathbf{r}_{N}\right),
\end{aligned}
$$

where $v_{i}^{P}$ is the Pauli potential of the $i$ th excited state. The Pauli energy of the pair-density-functional theory is analogous to that of the density-functional theory: the difference of the kinetic energy and a Weizsäcker-like expression [43] (constructed from the pair density instead of the density) [4].
The Pauli potential is the functional derivative of the Pauli energy with respect to the pair density. For a two-electron system the Pauli potential disappears.

The theory presented above is free from the $N$ representability problem [42,44-56]. However, the exact form of the Pauli potential is not known even for the ground state. Therefore, we have to find approximate expressions for numerical calculations. In constructing approximations [57] the $N$-representability problem might be important and should be taken in consideration.

The kinetic energy functional should be approximated, since the exact forms of the other terms in the total energy functional are known. One can try to use or generalize the ground-state approximate functionals. Higuchi and Higuchi developed a method to approximate the ground-state kinetic energy functional [58-60]. As their method is based on the constrained search technique, it can be extended to excited states. They use representable pair densities with a restriction of the searching area to the set of single Slater determinants. The kinetic energy functional $T_{s}$ gained by this procedure is, of course, different from the exact one and Higuchi and Higuchi [58-61] proposed several approximate forms for the difference $\Delta T$. Their technique can be extended to excited states by a further restriction of the searching area to those single Slater determinants that are orthogonal to the first $i-1$ states. Finally, one has to find adequate approximation for the difference $\Delta T$. Probably, one of the approximate forms proposed by Higuchi and Higuchi [58-60] will work for excited states, too. That will be the subject of further research.

\section{ACKNOWLEDGMENTS}

The work is also supported by the TAMOP 4.2.2.A$11 / 1 / \mathrm{KONV}-2012-0036$ project. The project is cofinanced by the European Union and the European Social Fund. Grant No. OTKA K 100590 is also gratefully acknowledged.
[1] P. Ziesche, Phys. Lett. A 195, 213 (1994); Int. J. Quantum Chem. 60, 149 (1996).

[2] P. Hohenberg and W. Kohn, Phys. Rev. 136, B864 (1964).

[3] A. Gonis, T. C. Schulthess, P. E. A. Turchi, and J. van Ek, Phys. Rev. B 56, 9335 (1997).

[4] Á. Nagy, Phys. Rev. A 66, 022505 (2002).

[5] Á. Nagy, in The Fundamentals of Electron Density, Density Matrices and Density Functional Theory in Atoms, Molecules and Solid State, edited by N. I. Gidopoulos and S. Wilson (Kluwer, Dordrecht, 2003), p. 79.

[6] F. Furche, Phys. Rev. A 70, 022514 (2004).

[7] A. K. Theophilou, J. Phys. C 12, 5419 (1978); N. Hadjisavvas and A. K. Theophilou, Phys. Rev. A 32, 720 (1985); A. K. Theophilou, Philos. Mag. B 69, 771 (1994); A. K. Theophilou and N. I. Gidopoulos, Int. J. Quantum Chem. 56, 333 (1995).

[8] E. K. U. Gross, L. N. Oliveira, and W. Kohn, Phys. Rev. A 37, 2805 (1988).

[9] Á. Nagy, Int. J. Quantum Chem. 69, 247 (1998); J. Phys. B 34, 2363 (2001).

[10] Á. Nagy, Int. J. Quantum Chem. 70, 681 (1998).
[11] Á. Nagy, in Electron Correlations and Materials Properties, edited by A. Gonis, N. Kioussis, and M. Ciftan (Kluwer, New York, 1999), p. 451.

[12] M. Levy and Á. Nagy, Phys. Rev. Lett. 83, 4361 (1999).

[13] Á. Nagy and M. Levy, Phys. Rev. A 63, 052502 (2001).

[14] Á. Nagy, M. Levy, and P. W. Ayers, in Theory of Chemical Reactivity, edited by P. K. Chattaraj (Taylor \& Francis, London, 2009), pp. 121-136.

[15] P. W. Ayers, M. Levy, and Á. Nagy, Phys. Rev. A 85, 042518 (2012).

[16] M. Levy, Proc. Natl. Acad. Sci. USA 76, 6062 (1979).

[17] E. H. Lieb, in Density Functional Methods in Physics, edited by R. M. Dreizler and J. da Providencia, NATO ASI Series, Series B (Plenum, New York, 1985), Vol. 123, p. 31.

[18] A. Görling, Phys. Rev. A 59, 3359 (1999).

[19] P. W. Ayers and M. Levy, Phys. Rev. A 80, 012508 (2009).

[20] F. Tasnádi and Á. Nagy, Chem. Phys. Lett. 366, 496 (2002).

[21] R. T. Sharp and G. K. Horton, Phys. Rev. 90, 317 (1953); J. D. Talman and W. F. Shadwick, Phys. Rev. A 14, 36 (1976); K. 
Aashamar, T. M. Luke, and J. D. Talman, At. Data Nucl. Data Tables 22, 443 (1978).

[22] J. B. Krieger, Y. Li, and G. J. Iafrate, Phys. Rev. A 45, 101 (1992); 46, 5453 (1992).

[23] Á. Nagy, Int. J. Quantum Chem. 99, 256 (2004).

[24] Á. Nagy, Adv. Quantum Chem. 42, 363 (2003).

[25] V. N. Glushkov and M. Levy, J. Chem. Phys. 126, 174106 (2007).

[26] M. Levy and J. P. Perdew, in Density Functional Methods in Physics, edited by R. M. Dreizler and J. da Providencia (Plenum, New York, 1985).

[27] R. Gaudoin and K. Burke, Phys. Rev. Lett. 93, 173001 (2004).

[28] V. Sahni, M. Slamet, and X. Y. Pan, J. Chem. Phys. 126, 204106 (2007).

[29] P. Samal, M. K. Harbola, and A. Holas, Chem. Phys. Lett. 419, 217 (2006).

[30] P. Samal and M. K. Harbola, J. Phys. B 38, 3765 (2005); 39, 4065 (2006).

[31] M. K. Harbola and P. Samal, J. Phys. B 42, 015003 (2009).

[32] M. Rahaman, S. Ganguly, P. Samal, M. K. Harbola, T. Saha-Dasgupta, and A. Mookerjee, Physica B 404, 1137 (2009).

[33] Md. Shamim and M. K. Harbola, J. Phys. B 43, 215002 (2010).

[34] M. Hemanadhan and M. K. Harbola, J. Mol. Struct.: THEOCHEM 943, 152 (2010).

[35] M. Hemanadhan and M. K. Harbola, Eur. Phys. J. D 66, 57 (2012).

[36] M. K. Harbola, M. Hemanadhan, Md. Shamim, and P. Samal, in Concepts and Methods in Modern Theoretical Chemistry, Vol. 1: Electronic Structure and Reactivity, edited by S. K. Ghosh and P. K. Chattaraj (CRC Press, Taylor \& Francis, Boca Raton, FL, 2013), p. 99.

[37] M. Hemanadhan, Md. Shamim, and M. K. Harbola, J. Phys. B 47, 115005 (2014).

[38] Á. Nagy and C. Amovilli, J. Chem. Phys. 121, 6640 (2004).
[39] Á. Nagy, J. Chem. Phys. 125, 184104 (2006).

[40] M. Levy, J. P. Perdew, and V. Sahni, Phys. Rev. A 30, 2745 (1984).

[41] Á. Nagy and C. Amovilli, J. Chem. Phys. 128, 114115 (2008).

[42] E. R. Davidson, Reduced Density Matrices in Quantum Chemistry (Academic Press, New York, 1976).

[43] C. F. Weizsäcker, Z. Phys. 96, 431 (1935).

[44] M. Levy and P. Ziesche, J. Chem. Phys. 115, 9110 (2001).

[45] A. J. Coleman, Rev. Mod. Phys. 35, 668 (1963); A. J. Coleman and V. I. Yukalov, Reduced Density Matrices: Coulson's Challange (Sringer-Verlag, New York, 2000); J. Cioslowski, Many-Electron Densities and Reduced Density Matrices (Kluwer/Plenum, New York, 2000).

[46] K. Husimi, Proc. Phys. Math. Soc. Jpn. 22, 264 (1940).

[47] P. O. Löwdin, Phys. Rev. 97, 1474 (1955).

[48] E. R. Davidson, Chem. Phys. Lett. 246, 209 (1995); Phys. Rev. A 1, 30 (1970).

[49] F. Sasaki, Phys. Rev. 138, B1338 (1965).

[50] J. K. Percus, J. Chem. Phys. 122, 234103 (2005); B. Liu and J. K. Percus, Phys. Rev. A 74, 012508 (2006).

[51] M. E. Pistol, Chem. Phys. Lett. 400, 548 (2004); 417, 521 (2006).

[52] P. W. Ayers and E. R. Davidson, Int. J. Quantum Chem. 106, 1487 (2006).

[53] P. W. Ayers, S. Gordon, and M. Levy, J. Chem. Phys. 124, 054101 (2006).

[54] P. W. Ayers, J. Math. Phys. 46, 062107 (2005).

[55] P. W. Ayers and M. Levy, J. Chem. Sci. 117, 507 (2006).

[56] C. Garnod and J. K. Percus, J. Math. Phys. 5, 1756 (1964).

[57] M. Higuchi and K. Higuchi, Phys. Rev. A 75, 042510 (2007).

[58] M. Higuchi and K. Higuchi, Physica B 387, 117 (2007).

[59] M. Higuchi and K. Higuchi, Phys. Rev. B 78, 125101 (2008).

[60] K. Higuchi and M. Higuchi, J. Phys. Condens. Matter 21, 064206 (2009).

[61] M. Higuchi and K. Higuchi, Comput. Theor. Chem. 1003, 91 (2013). 